$4{ }^{1}$ The University of Oklahoma, Department of Geography and Environmental Sustainability, 100

5 East Boyd St. SEC 650, Norman, OK 73019-1081, United States, Phone: (405) 325-9862,

6 jziolkowska@ou.edu

$7{ }^{2}$ The University of Oklahoma, Oklahoma Climatological Survey, 120 David L. Boren Blvd.,

8 Suite 2900, Norman, OK 73072, United States, Phone: (405) 325-9512, reuben.reyes@ ou.edu

$9 \quad *$ Corresponding author: Jadwiga R. Ziolkowska

\section{Geological and Hydrological Visualization Models for Digital Earth}

Representation

\author{
Jadwiga R. Ziolkowska $^{1 *}$ and Reuben Reyes ${ }^{2}$
}

11

12

10

\title{
Abstract
}

This paper presents techniques and interactive models for multi-dimensional analyses and geospatial visualization in virtual globes based on three application examples: 1) earthquakes around the world, 2) groundwater well levels in Texas, and 3) geothermal subsurface heat indexes in Texas.

While studies are known that represent multi-dimensional geospatial data points, we develop and suggest multi-dimensional models for virtual globes using KML and KMZ (compressed KML files) with a complete and static time series data set. The benefit of this approach for the user is the ability to view and analyze time-based correlations interactively over the entire time span in one instance, which is not possible with animated (dynamic) models.

The methods embedded in our models include: a) depth layer cueing within subsurface Earth visualization for a better orientation when maneuvering below the ground, b) a technique with Ternary Visual Shape Logic (TVSL) as a quick indicator of change over time, and c) different visual representations of multiple dimensions for the addressed case study examples. The models can be applied to a variety of problems in different disciplines, especially to support decisionmaking processes. 
Keywords: Multi-dimensional visualization, geospatial analysis, virtual globes, subsurface depth layer cueing, Ternary Visual Shape Logic (TVSL)

\section{Introduction}

In recent decades, computer graphics visualization and imaging have experienced a rapid growth and captured the interest of governments, scientists, and business representatives (Fox and Hendler, 2011). The idea of a virtual globe (a computer application allowing users to browse and search data projected on a cartographic representation of the Earth) emerged at the end of the last millennium (Bailey, 2010). The development of the XML-based markup language KML (Keyhole Markup Language) - open source, tag-based scripting language, interactive digital cartography became possible in all major virtual globes (NASA World Wind, Google Earth, Microsoft Virtual Earth, and ESRI's ArcGIS Explorer) (De Paor and Whitmeyer, 2011). Visualization techniques have been acknowledged to provide a powerful way to 'take advantage of human abilities to perceive visual patterns and to interpret them' (Andrienko et al., 2003; see also: Costabile and Malerba, 2003; Kopanak and Theodoulidis, 2003; Compieta et al., 2007).

Nowadays, most research studies apply two- and three-dimensional (2D and 3D) models (Rowe and Frank, 2011; Xu et al., 2010; Mao and Ban, 2013), especially for satellite imaginary (Zhang et al., 2007), 3D web graphics (Evans et al., 2014) or topography maps (Guo et al., 2009). Also, other multi-dimensional analyses using 4D models have been conducted over the years (Baum et al., 2007; Thomas et al., 1996; Li et al., 2011). However, most of those models and their standard spatial visualization in the existing geographical applications are not always adequate and sufficient for decision-support systems. New solutions are necessary that would include not only a static graphical view of the results, but that would also offer the possibility to interact with data 
50 in a spatial and temporal view (Compieta et al., 2007; Dransch et al., 2010; Shneiderman, 2002;

$51 \quad$ Van de Weghe et al., 2014).

52 The goal of this paper is to provide several geotemporal models that extend the current 53 knowledge in the field and meet the scientific and visualization needs as specified above. We 54 emphasize practical benefits of the models with three examples: earthquakes worldwide, 55 groundwater well levels and geothermal subsurface heat indexes in Texas.

56 Several studies are known representing multidimensional visualization examples. For instance,

57 Zhu et al. (2014a) developed a 3D KML model for visualizing borehole well information in 58 virtual globes. Zhu's et al. model, though valuable for research purposes, presents only an above59 the-surface perspective using height, size and color of the spheres to represent the same variable 60 (4D models). Also, Postpischl et al. (2011) developed 3D and 4D visualization models, using 61 KML beach balls for their earthquake visualization. However, also this study offers only a 3D or 62 4D perspective with a flat shape representation, not fully utilizing virtual globe environment.

63 The models presented here build upon and extend previous research in the field by: 1) presenting 64 both the above-the-surface perspective and below-ground subsurface perspective, and 2) using 65 the size of the sphere and color of the sphere independently of the geolocation, thus creating an 66 additional $5^{\text {th }}$ visualization dimension (5D model). The presented models can be used with 67 different virtual globes and on smartphone or mobile devices, which has not been reported in the 68 previous literature yet.

69 Nowadays, most 3D models are based on and apply specific visualization software that is not 70 always freely accessible to everyone, requires specialized graphic cards or hardware and/or is 71 difficult to use as it involves a long learning process. Virtual globe applications, used for this 72 research, do not have any special technical requirements in this regard and are capable of loading 
73 and rendering standard KML files. This feature allowed us to use KML files in a unique way to

74 develop models that would be accessible to anyone with very few limitations if any at all. The

75 interactive models overcome the current limitations in the field making visualization easily

76 accessible and interactively applicable for all computer users with Internet access, and thus

77 fostering scientific and educational learning.

78

79 Furthermore, the developed models can be classified as a visualization analytics tool that,

80 according to Andrienko et al. (2011), benefits from combining the strengths of human and

81 electronic data processing, and where by means of visualization and interactive visual

82 interfaces, humans and computers can converse and cooperate' (Keim et al. 2008). The models

83 also provide an exploration tool that, as advocated by Fox and Hendler (2011), 'scientists can use

84 throughout the research life cycle' not only to represent the final research results, but to look for

85 and interactively explore hidden patterns and relations that would not be detectable solely with

86 raw data sets.

87 The benefits, unique features, and contributions of the presented models to the multi-dimensional

88 visualization field can be summarized as follows:

89 1. They provide an interactive visualization platform that allows the user to adjust the input

90 parameters and specify the research needs more accurately. The platform is defined as any

91 virtual globe, e.g., Google Earth, NASA WorldWind, ArcGIS Explorer, ArcGIS Earth, and

92 Cesium.

93 2. They offer a multi-dimensional perspective in a single KML framework

94 3. They allow for spatio-temporal analyses covering both geographical location and the time $95 \quad$ variable without animation. 
4. They are freely accessible to the end user (open access models) and do not require advanced software or license access keys.

5. They can be used on any computer system: Windows, Linux, Apple, smartphones, or touch pads.

6. They can be easily transferred to and are compatible with other software packages like e.g. ArcGIS or QuantumGIS.

The models and an interactive data framework represent natural Earth measurements and humaninduced changes across geospatial time frames, at the global, national, and state levels. The techniques used in this paper will allow other researchers to view subsurface data with existing virtual globes to interact and see patterns in earthquake events, geothermal heat indexes, and groundwater well levels over time. The outcomes can be useful for further research in the field and to stakeholders to support decision-making processes related to earthquake emergency management, geothermal energy extraction, and sustainable water planning and management.

\section{Challenges for visual representation of complex data using static virtual globes}

This research applies virtual globes as currently the most comprehensive platform to represent multi-dimensional data. Despite their benefits, virtual globes still face several technological and methodological challenges. While we acknowledge those limitations briefly pointing them out in this section, we also provide solutions for the presented models and research examples.

For many decades, the 3D dimension has always been (and still is) a natural human part of comprehending real world information represented with several dimensions in virtual globes. Therefore, representing information from multi-dimensional data sets becomes the more challenging, the more dimensions we add to the visualization model. Furthermore, if the data is 
119 not grouped and/or categorized in a meaningful visual way, the usefulness of a model might be 120 lost. In addition, tools for manipulating data are not always readily available for specific types of

121 multi-dimensional data sets from different disciplines. Several programs are available for 122 displaying or converting data, like e.g. COLLADA, but more options are needed to provide a 123 comparison benchmark.

124 Another challenge is subsurface representation in virtual globes. Techniques of this kind are 125 rather recent, and they were not originally developed with the purpose of representing data below 126 the Earth's surface.

127 While 2D cross sections and pop-up planes or blocks that come above the surface of the Earth 128 are common (Dobson, 2012), they display solely pieces of subsurface as pop-up blocks. The 129 subsurface models presented with this paper represent the subsurface of the Earth as a whole and 130 is interactive meaning that it allows the user to fly-through and navigate under the Earth 131 subsurface.

132 Currently, when a user zooms into a highly populated cluster of data, orientation and location 133 may be lost. In cases like this more information about the subsurface surroundings is needed to 134 keep on track and better understand the information and orientation.

135 Moreover, currently animations are often used with temporal data in virtual globes. However, if 136 the time intervals are not set correctly, subtle information might be missed. Another issue with 137 animating large data sets arises when loading them into limited memory space, which may 138 prohibit viewing any of the data at all. In order to overcome this limitation, intelligent clustering 139 and reducing the size of the data is needed. Even the application of intelligent clustering 140 comparisons within a time-based static cluster will not insure a proper understanding of the data. 
141 Natural visual representation of the data as geometric shapes would considerably improve human

142 understanding of complex data sets.

143 Another potential limitation might be difficulty to present level of detail and memory 144 restrictions. However, using WebGL-based virtual globes like Cesium and OpenWebGlobe 145 (open source platforms) can alleviate this impediment.

\section{Methodology}

\section{$148 \quad 3.1 \quad$ Specifications of the KML/ KMZ models}

149 This paper develops new application methods and presents four types of models from different 150 scientific disciplines: geophysics, geology, and hydrology to represent earthquake events around 151 the globe (with a subsurface and above-the-surface representation - two separate models), 152 geothermal heat indexes, and groundwater well levels, respectively. Each model is stand-alone, 153 as it has unique application properties and features, while at the same time all models have 154 overlapping attributes. More specifically, all four models have two attributes in common: they 155 are georeferenced and represented with multiple visual dimensions. The various overlapping 156 shared attributes of the models are: the temporal dimension, application of depth layer cueing, 157 Ternary Visual Shape Logic (TVSL), and multiple visual dimensions. Table 1 summarizes the 158 model characteristics and applied functions.

160 [Table 1 here]

162 In addition to the new application methods presented here, this paper also builds upon several 163 standard computer visualization techniques that were commonly applied in the past in older 3D 
computer games. One of them is 'limited instances of a texture map' that were reuse multiple

165 times in the presented models to achieve higher memory efficiency. While this standard

166 technique has been used for years, it has not been widely incorporated into KML, as we suggest

167 in this paper for the applied geoscience examples. Furthermore, we also apply the 'natural

168 billboard effect' in virtual globes by using a 2D texture map of a sphere (Lawrol, 2005). This

169 technique allows for creating the effect of a true 3D sphere when viewing it from any side or

170 above and below. It also renders faster and uses less memory than representing a geometric 3D

171 sphere. In order to make the texture spheres look even more realistic we made use of the inherent

172 ' $z$ clipping' in virtual globes that avoids displaying pixelizaiton when zooming in close to the

173 texture map image.

174 To present practical application of those techniques and unique properties of the models, we 175 created a user-friendly visualization platform capable of 3D fly-throughs (visually conveying 176 meaningful information in a more comprehensive manner). This feature was facilitated by the 177 virtual globes and the developed KML code as a delivery format via the World Wide Web. The $1783 \mathrm{D}$ analysis space to output the results in the KML format was generated with the C++ 179 computing language. In all models the size and color of each sphere (data point) were used to 180 represent different data attributes. In some of the models an additional feature 'visual cues' has 181 been added to the environment (e.g., for the earthquake and geothermal models) to create a 182 subsurface wireframe with metadata that helps orientate and navigate through the subsurface. For 183 this purpose, depth-shaded vector outlines of counties and states at set intervals depths were 184 incorporated in the models, with varying gray values indicating different depths of earthquake 185 events or geothermal heat indexes. By clicking on any of the depth vector polygons, the object's 186 depth and the county/state can be visually identified. In the earthquake model, also translucent 
187 layers at set interval depths were added for depth layered cueing. Both the vector outlines layers

188 and the depth translucent layers can be turned on or off giving the user more visual options, as 189 needed.

\subsection{Methodology for subsurface visualization using depth layered cueing}

193 geothermal gradient model; both based on KMZ files. The visualization models for the 194 earthquake events use USGS data and Earth imagery data from NASA. In the presented model, 195 earthquakes are displayed at the global scale in the last 100 years (April 1914 - April 2014) with the magnitude range between 5.5 and $8.0>$, which generates a database with a total of 24,398 197 entries. Unlike previous studies on earthquake events, we represent the data set from the USGS 198 in a virtual globe in two different ways that represent an added value to the methodology and 199 literature in the field:

200 1) Subsurface visualization that applies two methods for depth layered cueing (providing the user 201 with a visual feedback about the spatio-temporal location, especially when using fly202 throughs):

a) Transparent Earth layers with changing transparency as a function of depth, and

b) Outline of countries at different depths intervals with each outline gray-scaled as a function of depth.

2) Above-the-surface visualization with elevation represented as a function of time (spatiotemporal analysis) and the earthquake depth represented as a color.

208 The presented visualization builds upon earthquake research presented by USGS (n.d.), while 209 also extending methodological knowledge in the field. The USGS models represent only a 4D 
210 perspective of earthquake events with flat circles on a flat globe surface. The models proposed

211 with this paper offer a unique perspective as they use spheres in the virtual globe environment,

212 and thus add another $\left(5^{\text {th }}\right)$ dimension to the earthquake visualization. This additional dimension

213 conveys a new piece of information and provides a more comprehensive analysis of earthquakes

214 compared to USGS models.

215 The multi-dimensional subsurface earthquake model in this paper combines the following 216 parameters: 1) latitude and longitude (x and y axes), 2) depth of the earthquakes in km ( $\mathrm{z}$ axis),

217 and 3) magnitude of the earthquakes represented both as the size and the color of the spheres. In

218 order to create the subsurface layer and present the multi-dimensional earthquake data below the

219 Earth's surface, the height of the Earth's surface was extended by $750 \mathrm{~km}$, which still allows for

220 the deepest earthquake in this model $(720 \mathrm{~km})$ to be viewed within this range. Next, an image

221 map of the Earth at an elevation of $750 \mathrm{~km}$ was set to be translucent, so that users could see

222 through the surface of the Earth down to the earthquake hypocenters (figure 1). The true depth

223 representation expressed through the $\mathrm{z}$ axis provides the user with a better understanding of 224 earthquake clusters and structures.

226 [Figure 1 here]

227

228 In 2012, De Paor et al. (2012) developed a COLLADA model representing a subsurface of the 229 Earth. Building upon De Paor et al.'s research, our models advance knowledge in this field as 230 they allow for fly-throughs and examining the respective geological events from a close 231 perspective and as a subsurface virtual Earth. The example KML models presented in this paper 232 do not require a specific version of Google Earth and can be used in any virtual globe supporting 
233 KML. To facilitate user orientation, outline vectors of all countries of the world were set up at 3 234 depths intervals for 3D fly-throughs. In addition, a semi-transparent image map of the Earth was 235 created at the depth of $300 \mathrm{~km}$ down as a user visual depth reference. Finally, we covered the 236 existing Google Earth with a blank black image to give the user more of a sense of depth, as 237 similarly done by De Paor et al. (2011). In a next step, the USGS earthquake data was converted 238 to KML for an interactive view in the virtual globe, while our compiled $\mathrm{C}++$ code allows for 239 representing the depth going down, starting at $750 \mathrm{~km}$ (simulated Earth's surface), and dropping 240 below the Earth's surface to explore and view earthquakes in 3D. This subsurface feature of the 241 model extends knowledge presented in the previous literature.

242 Other studies, e.g. by Zhu et al. (2016) and Zhu et al. (2014b) proposed volumetric subsurface 243 representation and developed a VisualCrust application and the SolidEarth system, respectively. 244 Our research uses a similar technique for the subsurface visualizations; however, we visualize 245 points (earthquakes) in subsurface to be viewed with virtual globes (3D viewers), which is 246 currently the most comprehensive way to view the models. The recent API depreciation 247 (embedding Google Earth within a browser) will not affect our models that can run on 3D 248 viewers independently of the browsers. We also chose to separate the subsurface representation 249 and the above-the-surface earthquake model (presented in section 3.3) due to the nature of our 250 data set visualized as points in the whole earth environment.

252 The geothermal indexes model uses the $\mathrm{x}$ and $\mathrm{y}$ variables to represent geolocation, while the 253 depth has been specified in a similar way as in the subsurface earthquake model described above. 254 This model uses color to represent heat gradient indexes curves across Texas and thus display an 255 additional (fourth) visual dimension (figure 2). Also in this case depth layer cueing was applied 
256 at three levels represented by vector outlines of the Texas counties. The vector layer color was

257 assigned as a function of depth (the deeper the representation, the darker the layer). In addition,

258 each county has assigned characteristics (e.g., the county name) and the depth of the geothermal

259 heat curves at each layer represented with the same method as in the earthquake model (here we

260 use counties instead of countries as in the earthquake model).

261

262 [Figure 2 here]

263

264 The combination of those techniques not only presents the user with visual depth layered cues,

265 but also provides a base for a reality check to verify the true geothermal depth while exploring

266 the model. The vector layers and translucent layers used for subsurface depth layered cueing can

267 be turned off or on by the user giving more flexibility in maneuvering through subsurface data

268 points.

269 The uniqueness of the subsurface earthquake and geothermal models results from the

270 combination of the 'depth layered cueing' technique with a subsurface KML model, making

271 them usable in any virtual globe.

272

$273 \quad$ 3.3 Methodology for above-the-surface visualization of earthquake events

274 To provide a comprehensive learning experience to the user and a different approach of 275 understanding data clustering, we developed an additional above-the-surface model for the 276 earthquake data set. For this visualization, the time of the earthquakes was incorporated as 277 elevation (old earthquakes are close to the surface, while most recent earthquakes are higher up 278 above the surface), thus adding one additional visual dimension to the previous subsurface 
279 model. The following model categories were included: 1) latitude, 2) longitude, 3) time variable 280 (date of the earthquake) represented with the height of the sphere above the Earth surface, 4)

281 magnitude of the earthquake represented with the size of the sphere, and 5) depth represented 282 with 10 color categories (the darker the color, the shallower the hypocenter of the earthquake; the 283 lighter the color, the deeper the hypocenter). The spheres are connected to the surface of the 284 Earth via a white vector line. This multi-dimensional earthquake model does not use depth 285 layering cueing techniques; therefore, the data set runs faster than the subsurface model, though a 286 longer rendering refresh time can be expected on low end computer systems (figure 3).

288 [Figure 3 here]

289

\subsection{Methodology of Ternary Visual Shape Logic as quick indicators of change}

291 For the groundwater well level model we invented the Ternary Visual Shape Logic (TVSL) 292 method that refers to the Russian computer Setun and its storage logic which used three states: 293 positive, negative, and neutral. The analogy of the three states from Setun represents three 294 recognizable visual states of groundwater levels discussed with this method: 1) cone shape 295 (groundwater level decrease), 2) inverted cone shape (groundwater level increase), and 3) no 296 discernible shape (no visible increase or decrease in groundwater well levels) (figure 4).

298 [Figure 4 here]

It needs to be emphasized that the TVSL is a visualization concept describing 'visual logic', not 301 a mathematical algorithm/ mathematical logic in the strict sense of the word. Through a visual 
302 representation of changing spheres humans are able to 'naturally' recognize patterns and trends

303 in data sets that would not be detectable instantly if Boolean algebra and operators were applied.

304 Thus, the TVSL concept has the capacity to make model results easily accessible to any user

305 with or without sophisticated knowledge in the computer and mathematical sciences. The

306 approach of representing patterns with a shape of multi-dimensional objects has proved to be

307 very successful for human recognition and data interpretation (Kourtzi et al., 2003; Jiang et al., 308 2008; Pizlo, 2007).

309 The TVSL has proven very useful in this study to visually detect statically relevant and 310 discernible well water changes over time for 12 consecutive years using spheres for each data 311 point representation and the height above the ground as the time variable, with the time intervals 312 grouped into three different height categories. The model used data on water wells level samples 313 in Texas with over 140,000 data points in 2003-2014. For the purpose of the anticipated 314 statistical analyses and in order to insure data conformity, the data set was further reduced to data 315 points from wells with at least one sample per year. The data was first grouped into three years 316 sections (2003-2006, 2007-2010, and 2011-2014), while four statistically relevant subgroups 317 within each of the three years sections were defined in a next step. This statistical subgrouping 318 reduced the data set to a manageable size and facilitated a smoother visualization process. Each 319 subgroup was color-coded, while also a sphere size was assigned to each of them in addition, 320 representing the same variable (well water level). The lowest water levels were assigned a light 321 blue color and a small sphere size, while the highest water levels were assigned a dark blue color 322 and a large sphere size. The statistical intervals were set in a way that the four subgroups range 323 from a small light blue sphere to a large dark blue sphere. If water levels were consistently going 324 down over years, the three spheres stacked one on top of the other would create a visual shape of 
325 a cone, with the highest sphere being the smallest one at the top of the cone (a witch's hat shape).

326 If the water levels were consistently going up over years, the sphere stack would display a cone

327 shape with the bigger sphere at the top of the cone (a frozen icicle shape). If water levels were

328 going up and down over the three time intervals, a cone shape would not be discernible.

3304 Results and discussion: Interactive geospatial multi-dimensional analysis of the three application examples

\subsection{Interactive global multi-dimensional earthquake models with depth layer cueing}

333 The subsurface layers can be easily recognized as translucent covers of the Earth. Earthquake 334 points are located either close to the upper layer (Earth surface) representing the shallow 335 earthquakes, or further down below the Earth's surface representing deeper earthquakes. The color and the size of the spheres represent the magnitude of the earthquakes. Figure 1 displays a 337 screen shot of earthquakes in Central and South America. In this part of the Earth, the 338 earthquakes have a higher magnitude and frequency as the Cocos plate and Nazca plate are 339 subducted along the coast. The model is interactive and can be adjusted to the user's needs by 340 turning on and off the model categories and features.

341 Figure 5 is another screen short of the subsurface model representing a view from the coast of 342 Philippines looking towards Japan at a depth of about 530 kilometers. The white lines dropping 343 from the surface of the Earth clearly substantiate the use of subsurface visualization and help to 344 exactly locate the earthquakes events as well as their frequency and magnitude. This allows the 345 end user to experience a true representation of the spatial distribution of earthquake events. Each 346 sphere provides additional information about the individual earthquake events giving the user the 347 option to view it interactively in a pop-up balloon window. Country layer outline vectors are also 348 interactive, thus providing additional information and orientation in the pop-up balloon windows. 
$350 \quad$ [Figure 5 here]

351

\subsection{Interactive multi-dimensional model for geothermal heat indexes in Texas with} depth layer cueing

354 The geothermal model presented here was derived from a data set with approximately 30,000

355 wells in Texas (figure 2), which represents only a small percentage of the total number of all 356 wells in the state. The well selection was determined by the data availability on wells with the 357 bottom hole temperatures. The spheres represent the geographical and approximate depth 358 location of the wells, while different sphere colors reflect different geothermal heat indexes that 359 are independent of the well depth. When clicking on the spheres, detailed information about the 360 heat index and the well are displayed, while due to its multi-dimensional format, the model 361 allows for fly-throughs at different depths. The presented geolocation of heat indexes can be 362 helpful both for supporting regional and statewide decision making processes as well as 363 educational curricula. Based on the cost of electricity, distance to larger cities (and demand for 364 energy), and depth of the geothermal heat layer to the surface, the model can be useful with 365 indicating a breakpoint for economic feasibility of building potential geothermal plants in the 366 respective locations across the state.

4.3 Interactive multi-dimensional model for well water levels in Texas with ternary visual shape

370 Figure 6 shows a screenshot from the multi-dimensional groundwater well model in a 371 neighborhood area of Lubbock in Texas. Each of the three spheres that are part of the Ternary 372 Visual Shape Logic are clickable and bring up a balloon information window with statistical 
373 information and includes a link to the original well data from the Texas Water Development 374 Board.

$376 \quad$ [Figure 6 here]

377

378 Due to a large data set, the model is densely populated and allows for an extensive analysis of 379 changes in the well water levels over time (figure 7). It is clear that in the analyzed time period 380 between 2003 and 2014 different measurements have been taken at different wells, with some 381 well water levels increasing and some decreasing over time. The model shows that water levels 382 in most of the wells have been decreasing, which has a potential to indicate the recent droughts 383 occurring in the state since 2011. The majority of the wells indicate a cone shape (witch's hat 384 shape) denoting a decrease of well water data. However, a minority of the wells are either 385 inconclusive to which direction the well water level changed over time. The number of wells 386 with increasing water levels represents only a small number that is displayed with the frozen 387 icicle cone shape. Due to the Ternary Visual Shape Logic, the model allows the user to 388 comprehend the results at one glace and determine where well water levels increased, decreased, 389 or remained unchanged. The model can be useful for educational purposes, but more specifically 390 for research and decision-making support, facilitating planning and efficient water management, 391 especially in times of drought. 
395 All models presented in this paper, including the subsurface representation of the earthquake 396 events and geothermal heat indexes can be viewed on any computer system and/or smartphone 397 (ipad, iphones or Androids), thus providing tools to a broader audience (figure 7-8).

$399 \quad$ [Figure 8 here]

$400 \quad$ [Figure 9 here]

401

\section{Conclusions and outlook}

403 With the increasing computerization the quality and advancement of visualization techniques

404 have been improving to better and more accurately describe real world problems. Yet, nowadays 405 most techniques use 2D and 3D visualization models that are still limited in their visualization 406 capabilities. Also, most multi-dimensional models use specialized licensed software that is not 407 available to everyone on the web and requires time to learn and utilize it.

408 Enhancing visualization techniques by new dimension features and providing an open access 409 model would help to make visualization broadly accessible and help solve relevant problems 410 more accurately and in a timely manner. To facilitate this development we developed interactive 411 multi-dimensional visualization models based on the virtual globes framework accessible freely 412 to anyone on the World Wide Web and easy to use for a targeted problem analysis.

413 The models can be used not only for research and teaching purposes, but also for supporting 414 decision-making processes. Interactive geospatial visual representation is crucial for the three 415 examples: earthquake, geothermal, and groundwater well levels. The subsurface earthquake and 416 geothermal visualization is unique by allowing an interactive view, while moving through 417 earthquake data sets below the Earth's surface with depth layered cues as guides and 3D 418 orientation. The Ternary Visual Shape Logic used in the model for Texas well water levels 
419 simplifies complicated large data sets used for statistical analyses into quick visual recognition of

420 geometric shapes. This method uses spheres to produce outlines of cones; however, other shapes

421 can be used instead to quickly and visually understand other types of complex and entangled

422 processes and data sets.

423 The presented techniques and models use multi-dimensional data and facilitate a better 424 understanding of and comprehensive interaction with different data dimensions. The models are 425 spatio-temporally oriented with the geographical location, color and size of the spheres 426 representing different data components. This provides a unique visualization, although the 427 current state of computer capacity can be limiting to a smooth and fast rendering process of 428 extremely large data sets like the presented earthquake data. However, according to the Moore's 429 law, computer capacity will improve in time along with advancements in graphic cards, thus 430 allowing for handling larger and more complicated data sets without any delays.

431 The main benefit of the geospatial interactive models is to deliver complicated data sets and a 432 dynamic rendering engine (here: a virtual globe) via the web and thus to make it available to 433 anyone to use at no cost by means of any modern computer system application (Windows, Mac, 434 Linux, and smartphones). Nowadays, virtual globes predominantly read KML and KMZ files 435 and thus offer a 3D visualization platform. We believe that other and more precise 3D 436 applications with the ability to read and render KML/ KMZ files will become available in the 437 course of time. They could provide a future platform to deal with larger and more complicated 438 data sets, create multi-dimensional stereographic displays and thus help the end user to 439 understand the visual data more easily and comprehensively.

440 We also anticipate future work in the field to develop virtual globes as a client and provide 441 dynamic processing on the server side computers. Similar work in this area has been done to 
442 move KML geometry elements via a user interface by Zhu et al. (2014c), while we also 443 appreciate promising features of Cesium for future work in this same area.

444 We propose the presented models to be used as an educational and research tool to better 445 analyze, present, and display research and teaching results. The models extend the current state 446 of knowledge in computer science and computer imaging and visualization. They can be 447 transferred to and applied to many other disciplines like medicine, epidemiology, social sciences, 448 engineering, and/or biology, to name just a few. They can also be used to extend the progress in 449 the field even further as the technology develops and allows for more complicated models in the 450 course of time.

451

452 Acknowledgment

453 This material is based on work supported by the National Science Foundation under Grant No. 454 OIA-1301789 and Oklahoma Climatological Survey (Mesonet). The authors acknowledge Bruce 455 L. Cutright and Harold Rogers for the geothermal data set.

457 Model access:

458 The models and an example video fly-through can be found here:

459 http://hitechmex.org/kml_examples/

460 The website is initially designed for the Editor and Reviewers, and will be refined upon 461 acceptance for publication. Thank you for your understanding. 
463

464

465

466

467

468

469

470

471

472

473

474

475

476

477

478

479

480

481

482

483

484

\section{References}

Andrienko G, Andrienko N, Keim D, MacEachren AM, and Wrobel S 2011 Challenging problems of geospatial visual analytics. Journal of Visual Languages and Computing 22: 251-256

Andrienko N, Andrienko G, and Gatalsky P 2003 Exploratory spatio-temporal visualization: an analytical review. Journal of Visual Languages and Computing special issue on Visual Data Mining 14(6): 503-541

Bailey J 2010 Virtual globes. In Encyclopedia of Geography (Warf B editor). Los Angeles, Sage Publications Inc. pp. 3528

Baum P, Yang DS, and Zewail AH 2007 4D Visualization of Transitional Structures in Phase Transformations by Electron Diffraction. Science 318: 788-92

Compieta P, Di Martino S, Bertolotto M, Ferrucci F, and Kechadi T 2007 Exploratory spatiotemporal data mining and visualization. Journal of Visual Languages and Computing 18: $255-279$

Costabile MF and Malerba D 2003 Special issue on visual data mining. Journal of Visual

Languages and Computing 14: 499-501

De Paor DG and Whitmeyer SJ 2011 Geological and geophysical modeling on virtual globes using KML, COLLADA, and Javascript. Computers \& Geosciences 37: 100-110

De Paor DG, Dordevic M, and Wild SC 2010 Using Google Earth to Visualize the Core, Mantle, and Crust in Four Dimensions. American Geophysical Union, Fall Meeting 2010

De Paor DG, Dordevic MM, and Wild SC 2011 Modeling Earth's Crust, Mantle, and Core with Google Mars and Google Moon. 2011 GSA Annual Meeting in Minneapolis 
Dobson KJ 2012 Visualizing subsurface geology with Google Earth. Cartographic Perspectives 72. WWW document, http://www.cartographicperspectives.org/carto/index.php/journal/article/view/cp72-peterson$\underline{\text { et-al/479 }}(5 / 7 / 2016)$

Dransch D, Köthura P, Schulte S, Klemann V, and Dobslaw H 2010 Assessing the quality of geoscientific simulation models with visual analytics methods - a design study. International Journal of Geographical Information Science 24(10): 1459-1479

Evans A, Romeo M, Bahrehmand A, Agenjo J, and Blat J 2014 3D graphics on the web: A survey. Computers \& Graphics 41: 43-61

Fox P and Hendler J 2011 Changing the Equation on Scientific Data Visualization. Science 331: 705

Global Water Intelligence (GWI) (DesalData.com) 2013 Market profile and desalination markets, 2009-2012 yearbooks and GWI website. WWW document, http://www.desaldata.com/ (02 July 2014)

Guo L, Jiang Y, Yang Y, and Tang H 2009 Research of 3D Representation Theory and Technology of Geographic Information. IEEE Computer Society: 326-330

Jiang Y, Boehler CN, Nönnig N, Düzel E, Hopf JM, Heinze HJ, and Schoenfeld MA 2008 Binding 3D Object Perception in Human Visual Cortex. Journal of Cognitive Neurosciences 20(4): 553-562

Keim D, Andrienko G, Fekete JD, Görg C, Kohlhammer J, and Melançon G 2008 Visual Analytics: Definition, Process, and Challenges. In: Kerren A, Stasko JT, Fekete JD, and North C (eds.) Information Visualization - Human-Centered Issues and Perspectives. Volume 4950 of LNCS State-of-the-Art Survey. Berlin, Springer, pp.154-175 
Kopanakis I and Theodoulidis B 2003 Visual data mining modeling techniques for the visualization of mining outcomes. Journal of Visual Languages and Computing 14(6): 543589

Kourtzi Z, Erb M, Grodd W, and Bülthoff HH 2003 Representation of the Perceived 3-D Object Shape in the Human Lateral Occipital Complex. Medicine \& Health Science \& Mathematics - Cerebral Cortex 13(9): 911-920

Lawrol OS 2005 Impostors for Parallel Interactive Computer Graphics. Dissertation, University of Illinois at Urbana-Champaign

Li W, Chen G, Kong Q, Wang Z, and Qian C 2011 A VR-Ocean system for interactive geospatial analysis and 4D visualization of the marine environment around Antarctica. Computers \& Geosciences 37: 1743-1751

Mao B and Ban Y 2013 Generalization of 3D building texture using image compression and multiple representation data structure. ISPRS Journal of Photogrammetry and Remote Sensing 79: 68-79

Pizlo Z 2007 Human Perception of 3D Shapes. Computer Analysis of Images and Patterns 4673 of the series Lecture Notes in Computer Science: 1-12

Postpischl L, Danecek P, Morelli A, Pondrelli S 2011 Standardization of seismic tomographic models and earthquake focal mechanisms data sets based on web technologies, visualization with keyhole markup language. Computers \& Geosciences 37: 47-56

Rowe T and Frank LR 2011 The Disappearing Third Dimension. Science 331: 712

Shneiderman B 2002 Inventing discovery tools: combining information visualization with data mining. Information Visualization 1(1): 5-12 
530 Thomas C, DeVries P, Hardin J, and White J 1996 Four-Dimensional Imaging: Computer

531 Visualization of 3D Movements in Living Specimens. Science 273(5275): 603-607

532 USGS n.d. Google Earth ${ }^{\mathrm{TM}}$ KML. Earthquake Hazards Program. USGS. WWW document,

533 http://earthquake.usgs.gov/earthquakes/feed/v1.0/kml.php (5/7/2016)

534 Van de Weghe N, De Roo B, Qiang Y, Versichele M, Neutens T, and De Maeyer P 2014 The

535 continuous spatio-temporal model (CSTM) as an exhaustive framework for multi-scale

536 spatio-temporal analysis. International Journal of Geographical Information Science 28(5):

$537 \quad 1047-1060$

$538 \mathrm{Xu} \mathrm{L}, \mathrm{Xu}$ Y, and Chow TWS 2010 PolSOM: A new method for multidimensional data

539 visualization. Pattern Recognition 43: 1668-1675

540 Zhang Y, Xie P, and Li H 2007 Multi-scale colour 3D satellite imagery and global 3D Web

541 mapping. IEEE Urban Remote Sensing Joint Event 2007: 1-8

542 Zhu L, Wang X, and Zhang B 2014a Modeling and visualizing borehole information on virtual

543 globes using KML. Computers \& Geosciences 62: 62-70

544 Zhu L, Sun J, Li C, Zhang B 2014b SolidEarth: a new Digital Earth system for the modeling and

545 visualization of the whole Earth space. Frontiers of Earth Science 8(4): 524-539

546 Zhu L, Wang X, Pan X 2014c Moving KML geometry elements within Google Earth.

$547 \quad$ Computers \& Geosciences 72: 176-183

548 Zhu L, Pan X, Sun J 2016 Visualization and dissemination of global crustal models on virtual 549 globes. Computers \& Geosciences 90 (Part A): 34-40

550

551 
552 Tables

553

554 Table $1 \quad$ Model specifications

\begin{tabular}{|l|c|c|c|c|c|}
\hline \multicolumn{1}{|c|}{ Model } & Georeferenced & Temporal & $\begin{array}{c}\text { Depth } \\
\text { Layer } \\
\text { Cueing }\end{array}$ & $\begin{array}{c}\text { Multi- } \\
\text { Ternary } \\
\text { Visual Shape }\end{array}$ & $\begin{array}{c}\text { Mumensional } \\
\text { (\# of } \\
\text { dimensions) }\end{array}$ \\
\hline $\begin{array}{l}\text { Earthquakes } \\
\text { (subsurface) }- \\
\text { World }\end{array}$ & Yes & No & Yes & No & Yes (4) \\
\hline $\begin{array}{l}\text { Earthquakes } \\
\text { (above-the- } \\
\text { surface)-World }\end{array}$ & Yes & Yes & No & No & Yes (5) \\
\hline $\begin{array}{l}\text { Geothermal } \\
\text { gradients - Texas }\end{array}$ & Yes & No & Yes & No & Yes (4) \\
\hline $\begin{array}{l}\text { Well water levels } \\
- \text { Texas }\end{array}$ & Yes & Yes & No & Yes & Yes (4) \\
\hline
\end{tabular}

555 Source: Authors' presentation

556

557 


\section{$558 \quad$ Figures}

559

560 Figure $1 \quad$ Subsurface earthquake map from above with translucent depth layers of Earth and 561 earthquakes events below, along Mexico, Central America and South America

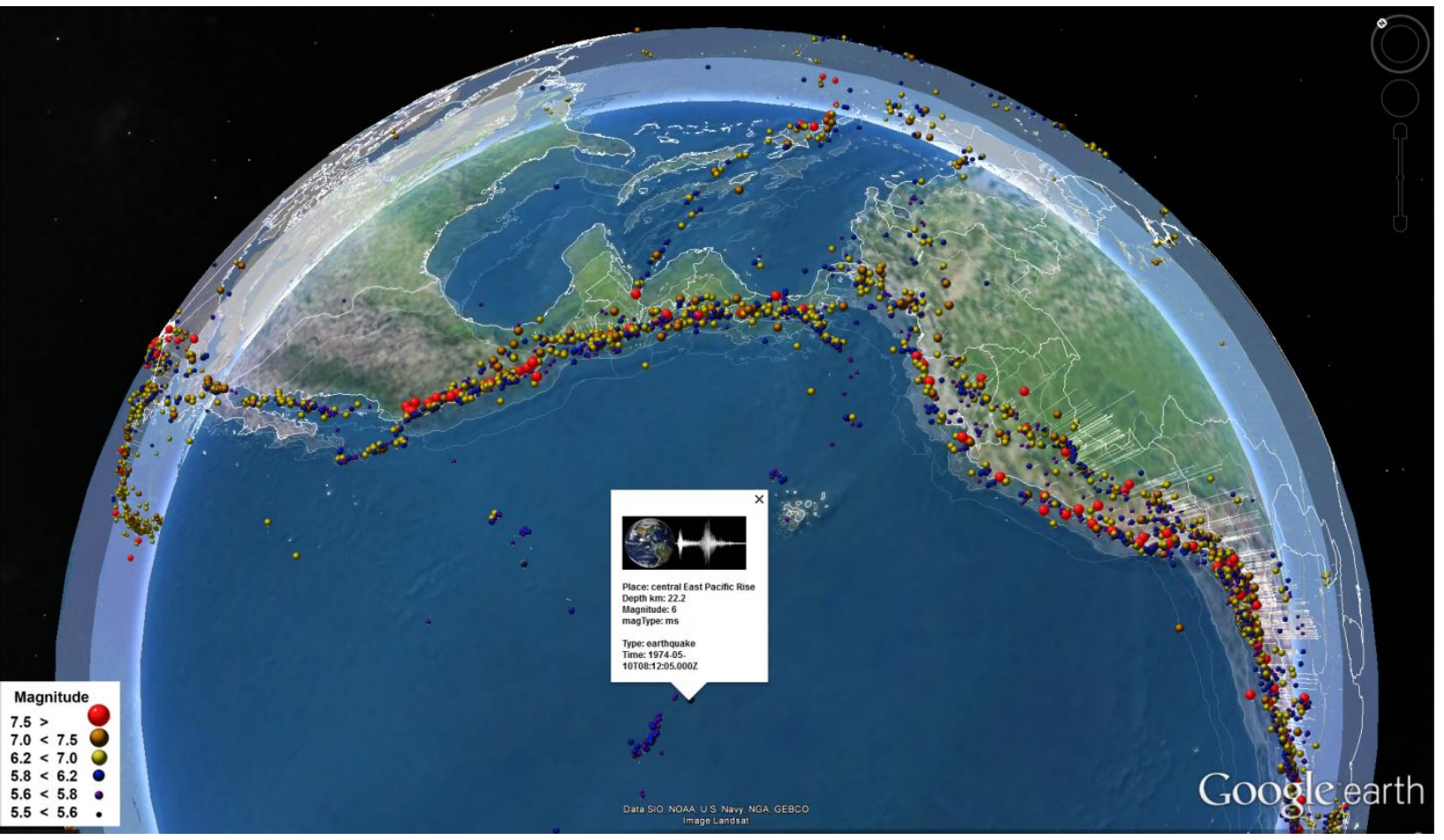

Source: Authors' calculation and presentation 
Subsurface perspective of geothermal heat indexes in Texas showing clusters in red and magenta in ArcGIS Earth

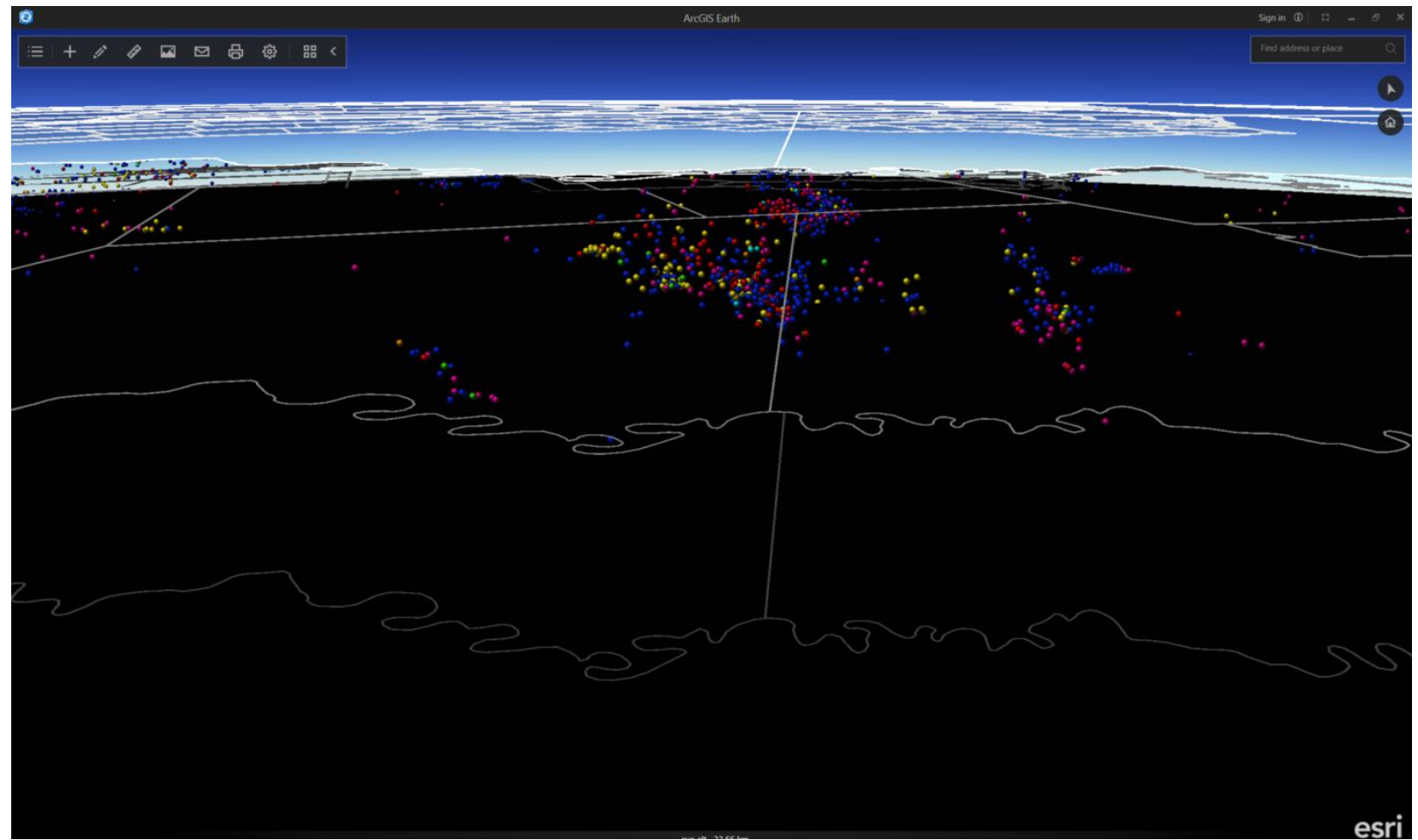

569 Legend: Texas county lines are displayed at three different levels (white at surface, gray at 570 10,000 feet below surface, and dark gray at 20,000 feet below surface).

571 Source: Authors' calculation and presentation

572 
573 Figure 3 Above-the-surface view of earthquakes off the coasts of Chile

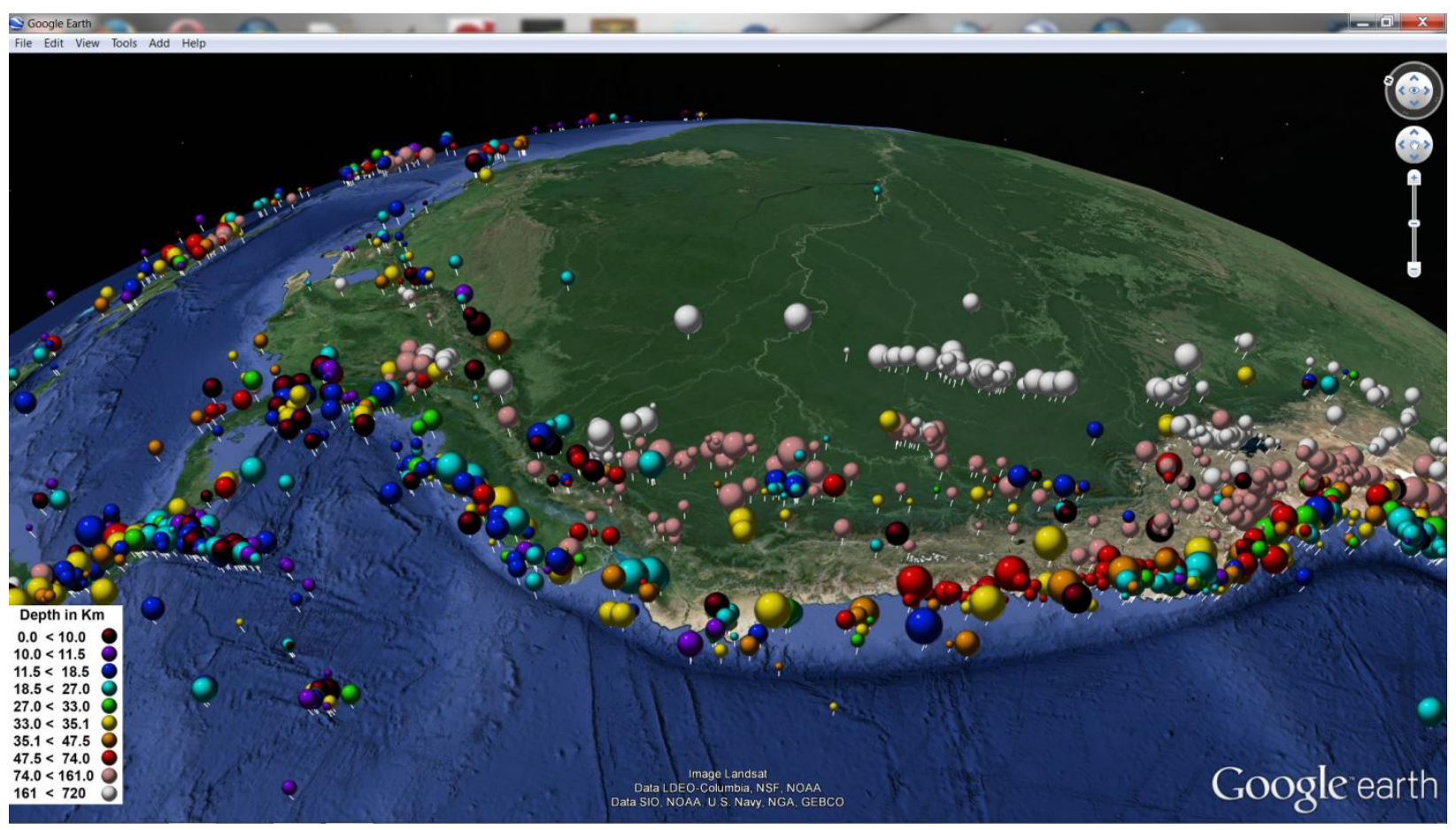

575 Source: Authors' calculation and presentation

576 
Figure 4 Ternary Visual Shape Logic diagram

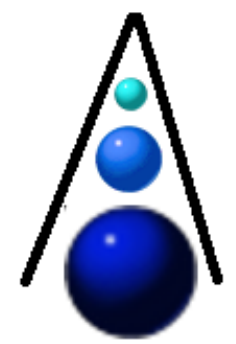

\section{discernible} decrease

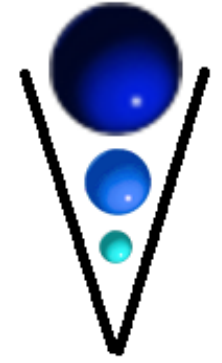

discernible increase

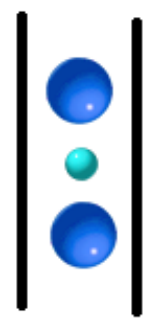

no discernible change

578

579 Source: Authors' calculation and presentation

580

581 
582 Figure $5 \quad$ View of deep earthquakes below the surface off the coasts of the Philippines and Japan at a depth of about 530 kilometers

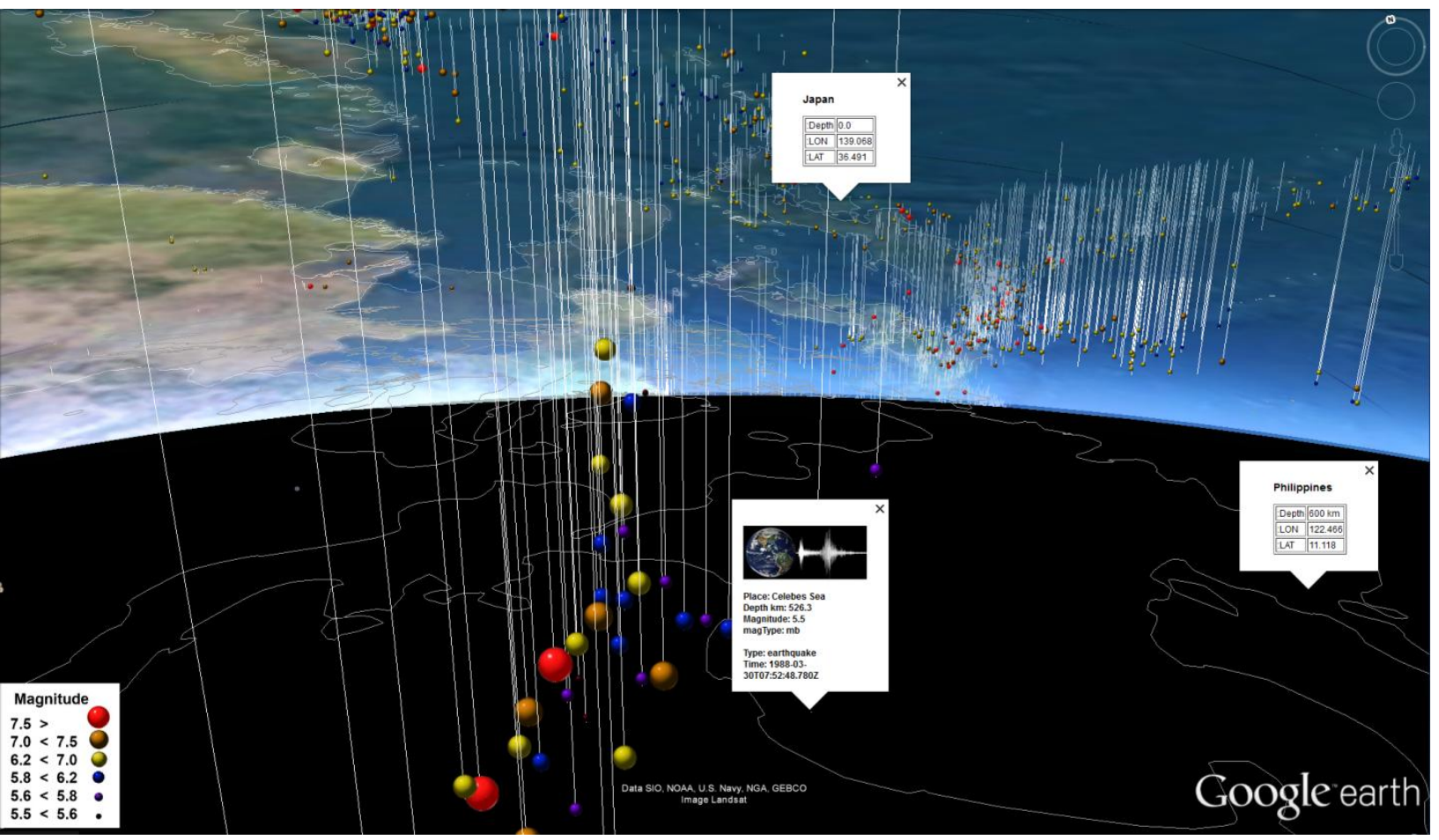

585 Note: White vectors connect the earthquake epicenter on the Earth's surface with the hypocenter $586 \quad$ spheres below the Earth's surface

587 Source: Authors' calculation and presentation 
589 Figure 6 Groundwater well levels in a multiple-dimension model in the region of 590 Abernathy in Texas

591

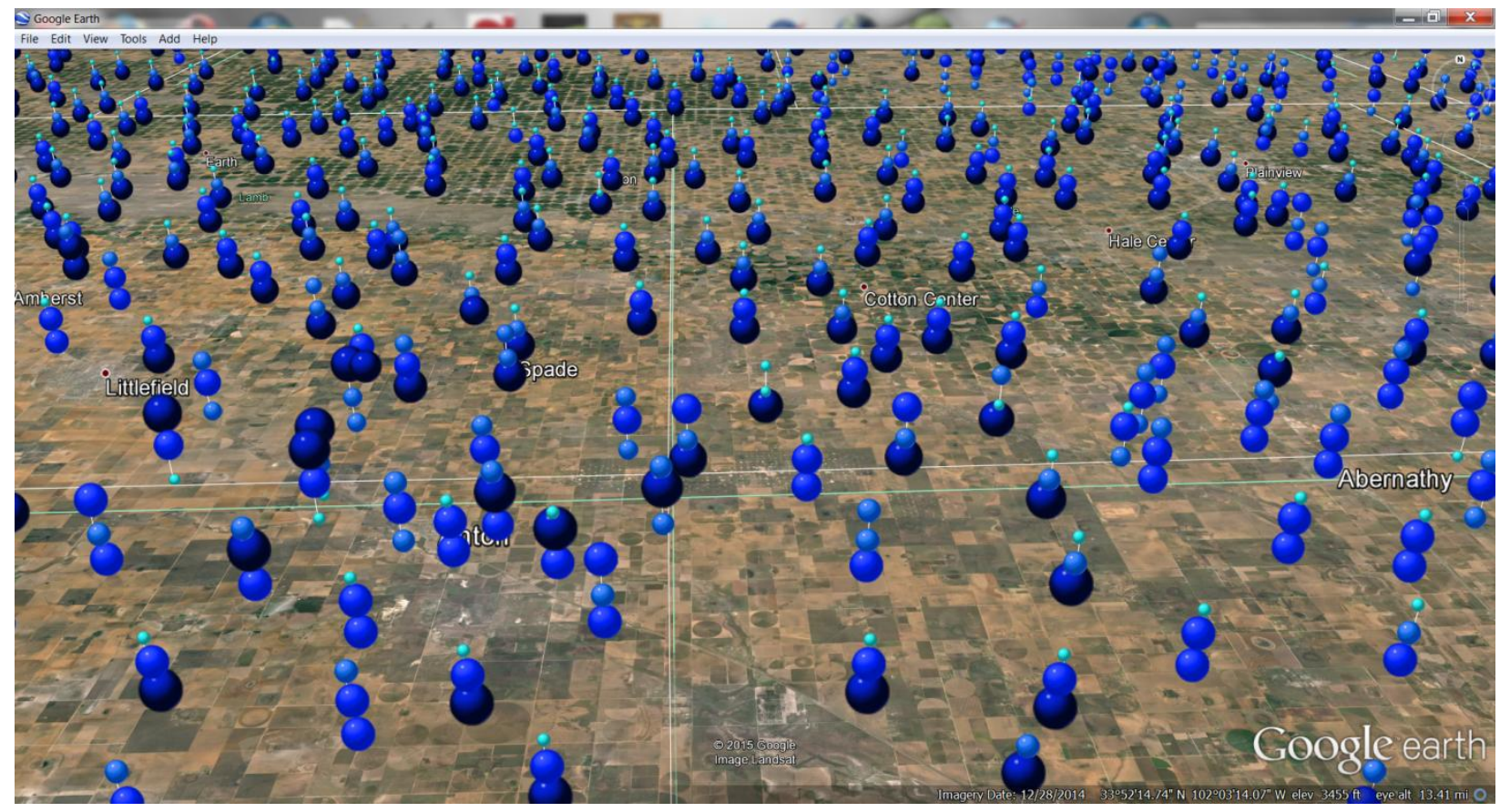

592 Source: Authors' calculation and presentation 
594 Figure $7 \quad$ Groundwater well levels in Texas in NASA World Wind KML Viewer

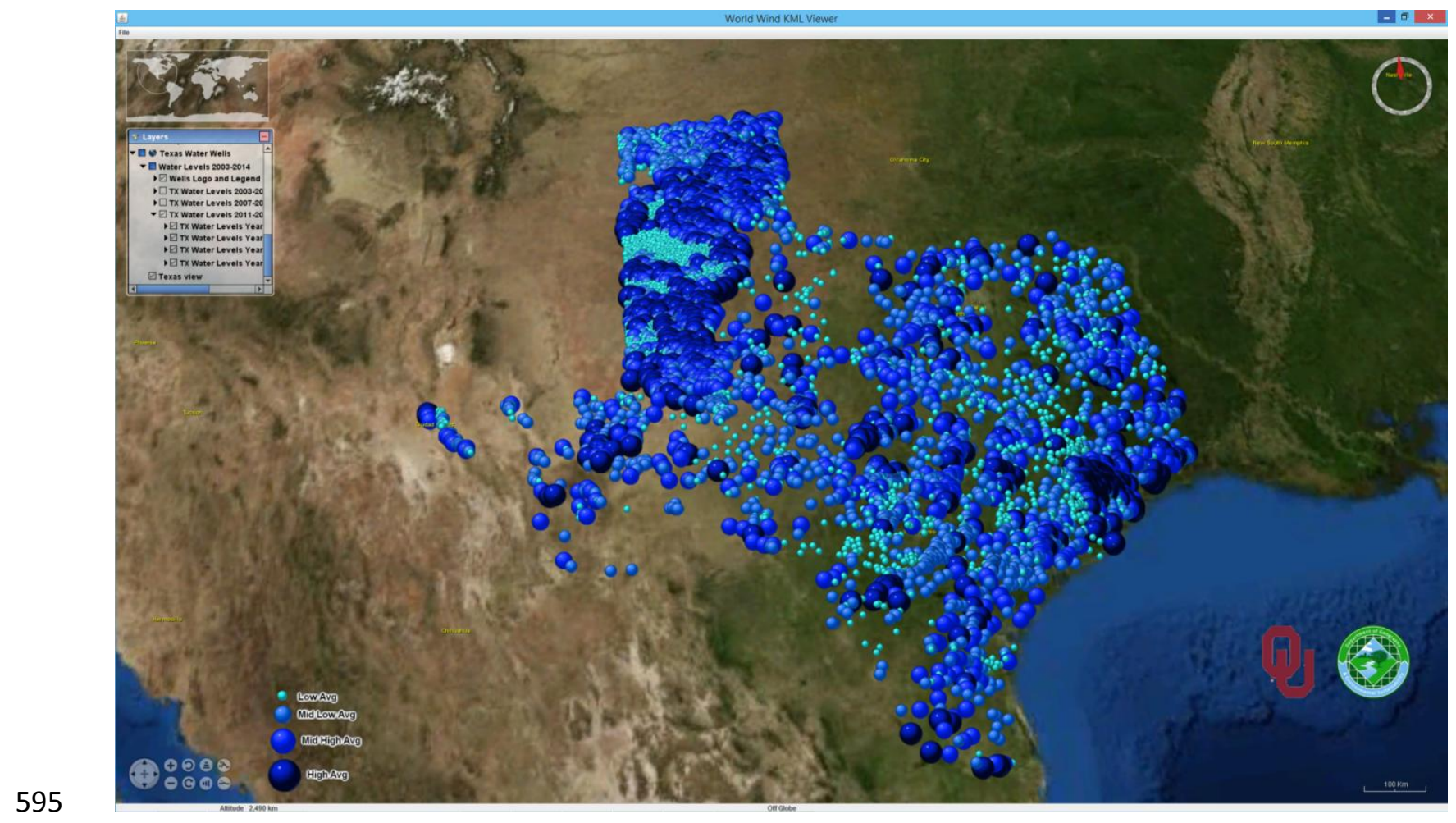

596 Source: Authors' calculation and presentation 
598 Figure 8 Geothermal heat indexes mapped with multiple dimensions across Texas on an 599 iphone

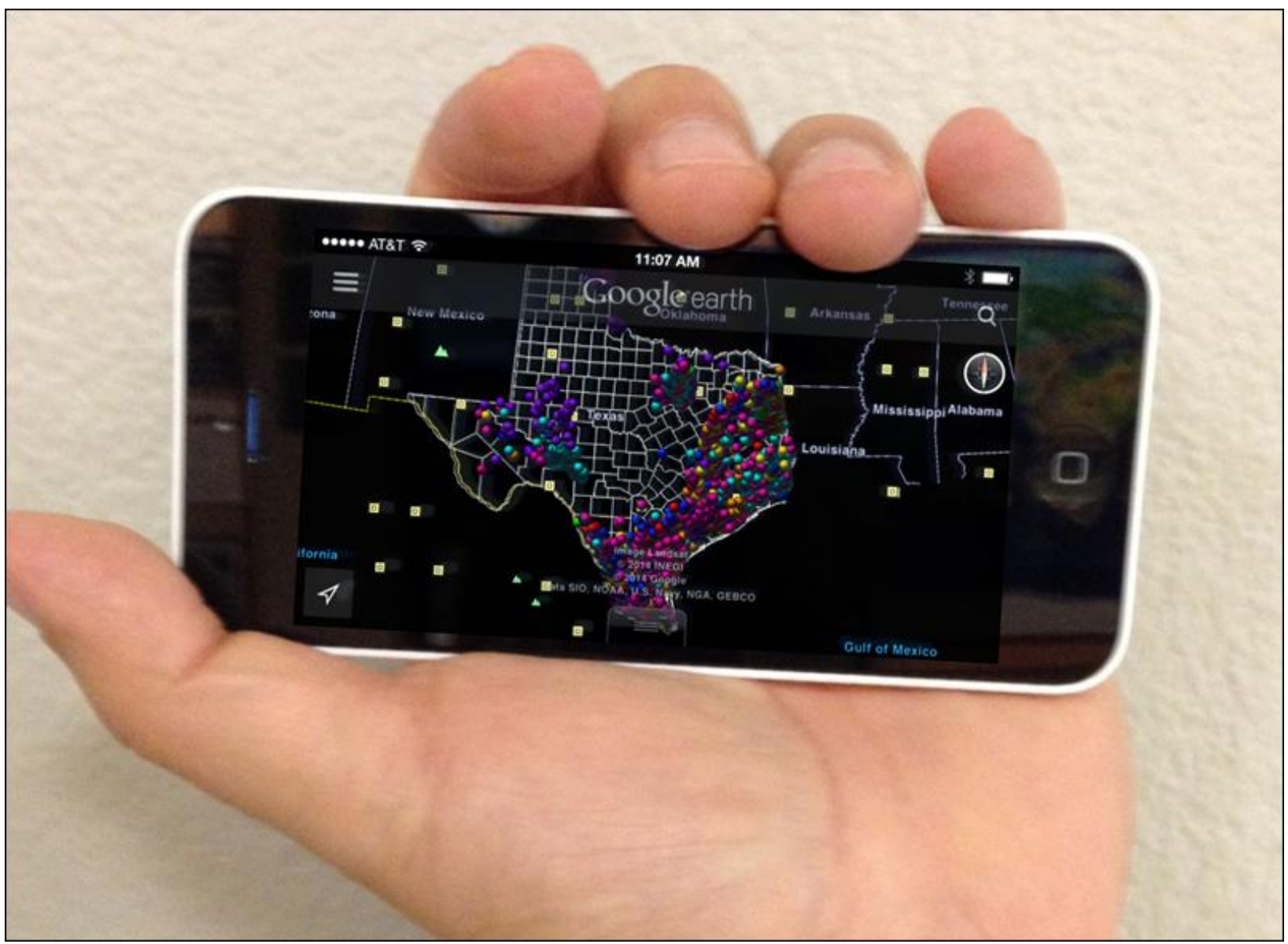

600

601 Source: Authors' calculation and presentation

602

603 


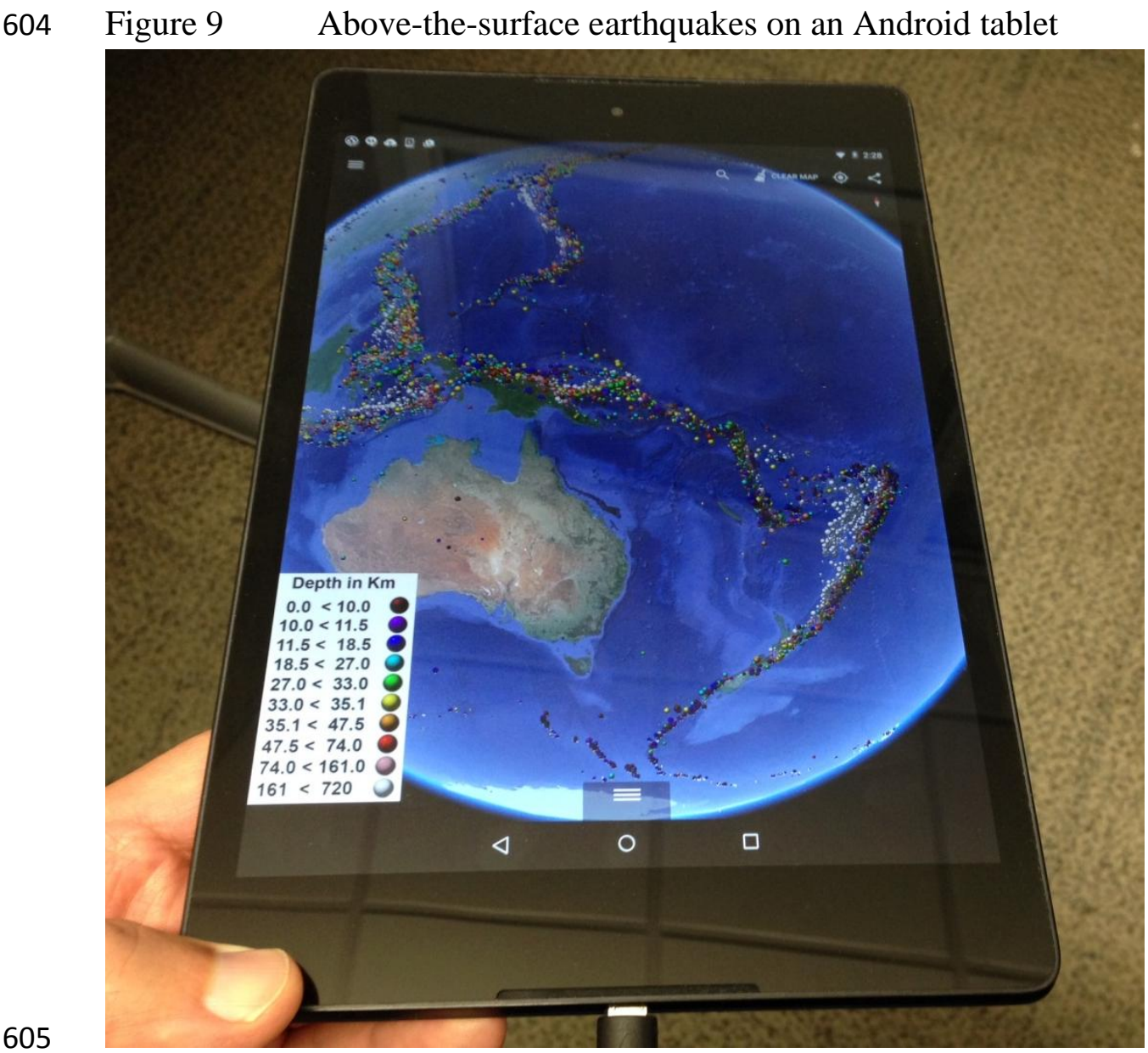

606 Source: Authors' calculation and presentation

607 
$609 \quad$ Figures

610 Figure 1 Subsurface earthquake map from above with translucent depth layers of Earth and

611 earthquakes events below, along Mexico, Central America and South America

612 Figure 2 Subsurface perspective of geothermal heat indexes in Texas showing clusters in $613 \quad$ red and magenta

614 Figure 3 Above-the-surface view of earthquakes off the coasts of Chile

615 Figure 4 Ternary Visual Shape Logic diagram

616 Figure 5 View of deep earthquakes below the surface off the coasts of the Philippines and 617 Japan at a depth of about 530 kilometers

618 Figure 6 Groundwater well levels in a multiple-dimension model in the region of 619 Abernathy in Texas

620 Figure $7 \quad$ Groundwater well levels in Texas in NASA World Wind KML Viewer

621 Figure 8 Geothermal heat indexes mapped with multiple dimensions across Texas on an $622 \quad$ iphone

623 Figure 9 Above-the-surface earthquakes on an Android tablet 